\title{
Analysis of Floquet formulation of time-dependent density-functional theory
}

\author{
Prasanjit Samal* and Manoj K. Harbolat \\ Department of Physics, Indian Institute of Technology, Kanpur U.P. 208016, India
}

\begin{abstract}
Floquet formulation of time-dependent density-functional theory is revisited in light of its recent criticism [Maitra and Burke, Chem. Phys. Lett. 359 (2002), 237]. It is shown that Floquet theory is well founded and its criticism has overlooked important points of both the Runge-Gross formalism and Floquet formulation itself. We substantiate our analysis by examples similar to that considered by Maitra and Burke.
\end{abstract}

${ }^{*}$ Electronic address: Fax:+91-512-259 0914 ; E-mail: dilu@iitk.ac.in

${ }^{\dagger}$ Electronic address: Fax:+91-512-259 0914 ; E-mail: mkh@iitk.ac.in 
Density functional theory (DFT) is a well established theory for the ground-states of Coulombic systems. It is conceptually simple and practically useful in various branches of physics, chemistry and material science. Based on the work of Hohenberg and Kohn (HK) [1] and Kohn and Sham (KS) [2], DFT has become a practical tool for the electronic structure calculation of atoms, molecules and solids [3]. Time-independent density functional formalism is also being extended to deal with excited-states [4, 5, 6, 7, 8, 9, 10, 11]. On the other hand, time-dependent phenomena are not accessible through traditional DFT. Time-dependent density functional theory (TDDFT) [12, 13, 14] is the generalization of the ground-state DFT to time-dependent problems. The development of TDDFT is relatively recent. The central result of modern TDDFT is a set of time-dependent Kohn-Sham equations which are structurally similar to the time-dependent Hartree-Fock equations but include in principle exactly all the many-body effects through a local time-dependent exchange-correlation potential. TDDFT allows access to lots of interesting and important phenomena which can't be easily dealt with in static DFT. It has become popular for studying atoms and molecules in intense laser fields [15], calculating the excitation spectra and oscillator strengths [16, 17], frequency dependent polarizabilities, hyperpolarizabilities [18] and optical response of solids, etc. [19]. TDDFT is especially very useful for quantum control problems [20]. This is because for intense laser fields where correlation effects are quite crucial, TDDFT appears to be the only way of studying the quantum dynamics of a many-electron system.

Initial steps towards the rigorous foundation of TDDFT were taken by Deb and Ghosh [12] and by Bartolotti [13]. They formulated and explored HK and KS type theorems for the time-dependent density. Each of these derivations, however, was restricted to a particular class of allowable time-dependent potentials (to potentials periodic in time). Because of the periodicity of the potential in time, Deb and Ghosh developed TDDFT with the Floquet formulation. Further in recent times Telnov and Chu [21, 22, 23, 24, 25] have developed approaches for the nonperturbative treatment of the strong field processes. These are the approaches which involve the transformation of the TDDFT equations into an equivalent time-independent generalized Floquet matrix eigenvalue problem. A variational 
perturbation method based on the Floquet theory has also been developed and applied [26] to study optical properties of atoms within TDDFT. This perturbative Floquet approach is suitable for the treatment of weak-field processes. A general proof of density to potential mapping $(\rho(\vec{r}, t) \rightarrow v(\vec{r}, t))$ for a time-dependent density was given by Runge and Gross [14]. Runge and Gross (RG) [14] formally established the TDDFT by showing that for a given initial state the evolving density $\rho(\vec{r}, t)$ uniquely determines the corresponding time-dependent potential $v(\vec{r}, t)$. Two features of RG formalism are noteworthy: one that the time-dependent wavefunction for a given density has functional dependence on both the density as well as the initial wavefunction $\Psi_{0}$. Secondly the formalism is valid for potentials that are Taylor series expandable at the initial time $t=t_{0}$. This makes the formalism applicable to suddenly switched-on potentials but not to the adiabatically switched-on potentials.

Like in the case of static DFT, in TDDFT also an interacting many-electron system is mapped onto a fictitious non-interacting Kohn-Sham system with the same time- dependent density as the interacting system. In Floquet formulation [12, 13] the Kohn-Sham system is developed in terms of the periodic time-dependent density only. On the other hand, in the the RG formalism the Kohn-Sham wavefunction is a functional of both the time-dependent density and an initial state [27, 28]. The uniqueness of the KS system follows from the one-to-one $\rho(\vec{r}, t) \rightarrow v(\vec{r}, t)$ mapping. However, in a recent work [29] by Maitra and Burke it has been suggested that a KS system within the Floquet formalism is not unique. The non-uniqueness of the KS system has been attributed to its functional dependence on the initial state. Further, they suggest that the Hohenberg-Kohn like theorem [12] may not exist in Floquet theory of TDDFT because the "ground Floquet state" cannot be defined properly. This appears to have created some doubts [30] about the Floquet theory in TDDFT. It is the purpose of this paper to analyze the Floquet theory of TDDFT and show that it is perfectly valid. The present analysis also brings out subtle differences between the RG and Floquet theory and shows the two formulations to be distinct and founded on two different principles. As such Floquet theory of TDDFT cannot be thought of as a particular case of the RG theory and the RG arguments can't be applied to Floquet formulation. We begin with a short description of the foundations of Floquet and RG theory in TDDFT. 
For a time-dependent (TD) Hamiltonian $\hat{H}(t)$ periodic in time, i.e.

$$
\hat{H}(t)=\hat{H}(t+\tau)
$$

where the period $\tau$ is positive with $\omega=\frac{2 \pi}{\tau}$, the TD Scrödinger equation (atomic units are used throughout)

has solutions [31, 32] of the form

$$
\left[\hat{H}(t)-i \frac{\partial}{\partial t}\right] \Psi_{n}(\vec{r}, t)=0
$$

$$
\Psi_{n}(\vec{r}, t)=\phi_{n}(\vec{r}, t) e^{-i \varepsilon_{n} t} ; \phi_{n}(\vec{r}, t)=\phi_{n}(\vec{r}, t+\tau)
$$

where the time-periodic functions $\phi_{n}(\vec{r}, t)$ are termed as the quasienergy eigen states (QES) and $\varepsilon_{n}$ are referred to as the the quasienergy eigenvalues. The steady-state wavefunctions $\phi_{n}(\vec{r}, t)$ are solutions of

$$
\left[\hat{H}(t)-i \frac{\partial}{\partial t}\right] \phi_{n}(\vec{r}, t)=\varepsilon_{n} \phi_{n}(\vec{r}, t)
$$

where $\phi_{n}(\vec{r}, t)$ are square integrable and $\varepsilon_{n}$ are real numbers. The quasienergies, $\varepsilon_{0}, \varepsilon_{1}, . ., \varepsilon_{i}, \ldots$ are defined 'modulo $(\omega)$ '. However, they can be ordered in increasing order $\varepsilon_{0}, \varepsilon_{1}, \ldots$ by making sure that as the strength of the applied time-dependent potential goes to zero, each quasi-energy go to its unperturbed counterpart [33]. Thus as long as $\omega$ is not one of the resonant frequencies, there exist the "ground-state energy" $\varepsilon_{0}$ and "excitedstate energies" $\varepsilon_{1}, \ldots, \varepsilon_{n}$ [33] in the steady-state formalism (see Appendix A for details). The theory of solutions in time-periodic Hamiltonian can be thought of like the stationary-state theory but in an extended Hilbert space $(R+T)$ that includes in addition to the space dependent function, time-periodic functions also. The operator

$$
\mathcal{H}(t)=\hat{H}(t)-i \frac{\partial}{\partial t}
$$

is called the Hamiltonian for the steady states in the composite Hilbert space, which resembles in many way the Hamiltonian for bound-states. The scalar product in this space is defined as 


$$
\{\langle\phi \mid \psi\rangle\}=\frac{1}{\tau} \int_{0}^{\tau} d t \int \phi^{*}(\vec{r}, t) \psi(\vec{r}, t) d \vec{r}
$$

i.e. in addition to the space integral, an integral over time is also taken. Here the curly brackets ' \{\} ' indicate the time average over a period. Thus the quasienergy functional is given by

$$
\varepsilon_{n}\left[\phi_{n}\right]=\left\{\left\langle\phi_{n}|\mathcal{H}(t)| \phi_{n}\right\rangle\right\}
$$

We point out - and this is important from TDDFT point of view - that the steady-state solutions are obtained by an adiabatic switching of the periodic potential. Finally applying the variational principle, each state $\phi_{n}(\vec{r}, t)$ can also be obtained [32] by making the expectation value $\left\{\left\langle\phi_{n}\left|\hat{H}(t)-i \frac{\partial}{\partial t}\right| \phi_{n}\right\rangle\right\}$ stationary. The stationarity principle becomes a minimum principle for the "ground-state" (Here the "ground-state" refers to a steady-state having the lowest quasienergy.) by assuming completeness of the set $\left\{\phi_{n}\right\}$ over the $(R+T)$ space. The general proof of the minimum principle for the ground-Floquet state is given in Appendix A.

Using the minimum property of the ground-state quasienergy functional a HohenbergKohn like theorem can be proved [12] for time-dependent periodic densities also. We reproduce the proof below. Let us consider the nondegenerate ground-state of a system characterized by the Hamiltonian $\hat{H}(t)$. Suppose there exist two different Floquet ground-states $\phi$ and $\phi^{\prime}$ corresponding to the steady state Hamiltonians $\mathcal{H}$ and $\mathcal{H}^{\prime}$, or more specifically corresponding to the external potentials $v(\vec{r}, t)$ and $v^{\prime}(\vec{r}, t)$, both of which give rise to the same TD density $\rho(\vec{r}, t)$. Using the minimal property for the ground-state we get

$$
\varepsilon^{\prime}<\varepsilon+\frac{1}{\tau} \int_{t_{1}}^{t_{2}} d t \int\left[v^{\prime}(\vec{r}, t)-v(\vec{r}, t)\right] \rho(\vec{r}, t) d^{3} r .
$$

Similarly for the ground-state of the Hamiltonian $\mathcal{H}$

$$
\varepsilon<\varepsilon^{\prime}+\frac{1}{\tau} \int_{t_{1}}^{t_{2}} d t \int\left[v(\vec{r}, t)-v^{\prime}(\vec{r}, t)\right] \rho(\vec{r}, t) d^{3} r .
$$

Adding Eqs. (8) and (91) results in the absurdity,

$$
\varepsilon+\varepsilon^{\prime}<\varepsilon^{\prime}+\varepsilon
$$


Hence two different potentials $v(\vec{r}, t)$ and $v^{\prime}(\vec{r}, t)$ cannot give the same density $\rho(\vec{r}, t)$, which implies that the time-dependent potential $v(\vec{r}, t)$ is a unique functional of the time-dependent density $\rho(\vec{r}, t)$. Accordingly, $\mathcal{H}, H$ and $\phi$ and indeed any "ground-state" property are all unique functional of $\rho(\vec{r}, t)$. Thus for Floquet states the HK theorem is [12] : "the density corresponding to the ground-state quasienergy of a time-periodic Hamiltonian determines the corresponding external time-periodic potential $v_{\text {ext }}(\vec{r}, t)$ uniquely". For example in perturbation theory up to the second order in energy, $E^{(2)}$ is minimum [26] with respect to $\rho^{(1)}$ for frequencies less than the first excitation frequency (i.e. $\omega<\omega_{10}=E_{1}-E_{0}$, where $E_{0}$ and $E_{1}$ are the unperturbed ground and excited-state energies). Thus in the linear response regime, the theorem would apply to $\rho^{(1)}$ up to frequency $\omega<\omega_{10}$. This theorem is then the foundation of Floquet theory of TDDFT [21, 22, 23, 24, 25, 26]. We point out that the theory does not have any initial state dependence but requires adiabatic switching of the applied external potential. Assuming the $v$-representability, an equivalent Kohn-Sham system for an $N$-electron system can also be developed.

As pointed out earlier, in RG theory [14] the proof of the density-to-potential mapping for time-dependent systems is based directly on the TD Schrödinger equation. In this theory, it is assumed that the time-dependent potential $v(\vec{r}, t)$ is turned on at a particular time $t=t_{0}$ and all systems are taken to evolve from the same initial state wavefunction $\Psi\left(t_{0}\right)=\Psi_{0}$. It is then shown that if the potential has a Taylor series expansion around the initial time $t=t_{0}$, then the time-dependent density $\rho(\vec{r}, t)$ determines the potential $v(\vec{r}, t)$ uniquely. In turn $\Psi(\vec{r}, t)$ is also determined by $\rho(\vec{r}, t)$. Thus the RG theory is quite distinct from the Hohenberg-Kohn theorem or its steady-state DFT counterpart (Deb-Ghosh theorem proved above), which are based on the minimization of energy (or quasienergy in the Floquet formalism). Notice that because of the Taylor series expansion requirement of $v(\vec{r}, t)$, RG theorem is applicable to suddenly switched-on potentials but not to adiabatically switched-on potentials. Table I gives a comparison of the two theories.

It is clear from the comparison given in Table I that the two theories are quite distinct and their domain of application is also different. As such Floquet formulation of TDDFT 
TABLE I: Comparison of Floquet theory and RG theory of TDDFT

\begin{tabular}{|c|c|}
\hline Floquet Theory & RG Theory \\
\hline $\begin{array}{l}\text { - Based on the minimum principle } \\
\text { of the ground-state energy. } \\
\text { - Steady-state is reached by } \\
\text { adiabatic switching of the } \\
\text { time-periodic potential. } \\
\text { - } v(\vec{r}, t) \text { is functional of } \rho(\vec{r}, t) \text { only. }\end{array}$ & $\begin{array}{l}\text { - Based on the evolution of } \\
\text { wavefunction from } t=t_{0} \text {. } \\
\text { - Wavefunctions are obtained } \\
\text { from potentials analytic a } t=t_{0} \text {. } \\
\text { Adiabatic switching is ruled out. } \\
\text { - } v(\vec{r}, t) \text { is functional of } \rho(\vec{r}, t) \text { as } \\
\text { well as } \Psi_{0} \text {. }\end{array}$ \\
\hline
\end{tabular}

[12, 13, 21, 22, 23, 24, 25, 26] is not a particular case of the RG theory. In light of this we now analyze the paper by Maitra and Burke [29] and then go on to comment on what does the distinction between the Floquet and the RG theory mean in the context of calculation of quantities like polarizabilities $\alpha(\omega)$ and excitation energies etc. using TDDFT.

Maitra and Burke consider the example of two non-interacting electrons in a onedimensional harmonic oscillator potential subject to an adiabatically switched-on timedependent potential periodic in time. Thus the Hamiltonian they consider is

$$
H=-\frac{1}{2} \frac{d^{2}}{d x^{2}}+\frac{1}{2} \omega_{0}^{2} x^{2}+\lambda x \operatorname{Sin}(\omega t)
$$

Due to the lack of many exactly known analytic solutions of time-dependent systems, we also consider the same system. The QESs for the above mentioned Hamiltonian are known analytically [34] to be

$$
\phi_{n}(x, t)=\psi_{n}(\bar{x}(t)) e^{\{i(\omega A x \operatorname{Cos}(\omega t)+\alpha(t)\}}
$$

where $\psi_{n}$ are the eigenstates of the static Harmonic oscillator and $\bar{x}(t)=x-A \operatorname{Sin}(\omega t)$. The parameters of the wavefunction are given as

$$
A=\frac{\lambda}{\omega^{2}-\omega_{0}^{2}}
$$


and

$$
\alpha(t)=\lambda^{2} \frac{\left\{\frac{\operatorname{Sin}(2 \omega t)}{8}+\operatorname{Cos}(\omega t)-1-\omega^{2} \operatorname{Cos}(\omega t) \times \frac{\operatorname{Sin}(\omega t)}{\left(\omega^{2}-\omega_{0}^{2}\right)}\right\}}{\omega\left(\omega^{2}-\omega_{0}^{2}\right)}
$$

The corresponding quasienergy for the QES $\phi_{n}(x, t)$ is

$$
\varepsilon_{n}=\left[\left(n+\frac{1}{2}\right) \omega_{0}+\frac{\lambda^{2}}{4\left(\omega^{2}-\omega_{0}^{2}\right)}\right] \operatorname{modulo}(\omega)
$$

We first point out that the quasienergies have been expressed in such a manner that as $\lambda \rightarrow 0$, the energy eigenvalues go to their respective time-independent eigenvalues. Thus energy defined 'modulo $(\omega)$ ' does not create any difficulty. Secondly, as long as $\omega \neq \omega_{0}$, there is clearly a well defined "ground-state" in these solutions. Thus $\varepsilon_{0}=\frac{\omega_{0}}{2}+\frac{\lambda^{2}}{4\left(\omega^{2}-\omega_{0}^{2}\right)}$ represents the "ground-state" energy of the system, whereas the other energies are the "excited-state" energies.

Maitra and Burke consider a singlet state with one electron occupying the ground-state (i.e. $n=0$ ) and the other the first excited-state (i.e. $n=1$ ) quasienergy orbitals. This gives the density of the system to be

$$
\rho(x, t)=\sqrt{\frac{\omega_{0}}{\pi}}\left(1+2 \omega_{0} \bar{x}(t)^{2}\right) e^{-\omega_{0} \bar{x}(t)^{2}}
$$

Now they generate the same density by another system with a different periodic potential. For doing this they consider a Floquet state which is also a spin singlet but with one doubly occupied steady state. Thus

$$
\tilde{\Phi}\left(x_{1}, x_{2}, t\right)=\tilde{\phi}\left(x_{1}, t\right) \tilde{\phi}\left(x_{2}, t\right) e^{2 i \tilde{\varepsilon} t}
$$

with

$$
\tilde{\phi}(x, t)=\sqrt{\frac{\rho(x, t)}{2}} e^{i \beta(x, t)},
$$

where $\beta(x, t)$ is a real time-periodic function, $\beta(x, t+\tau)=\beta(x, t)$. By inverting the TD Schrödinger equation one will have a different potential

$$
\tilde{v}(x, t)=\frac{1}{2} \frac{\tilde{\phi}^{\prime \prime}(x, t)}{\tilde{\phi}(x, t)}+i \frac{\dot{\tilde{\phi}}(x, t)}{\tilde{\phi}(x, t)}+\tilde{\varepsilon}
$$


In this $\tilde{\varepsilon}$ is the orbital quasienergy for the second Floquet state. The function $\beta(x, t)$ is determined by assuming the potential and quasienergy to be real. Now the new potential and phase for the second Floquet state are given by

$$
\begin{aligned}
\tilde{v}(x, t)= & \frac{1}{2} \omega_{0}^{2} \bar{x}(t)^{2}\left\{1-\frac{4}{\left(1+2 \omega_{0} \bar{x}(t)^{2}\right)^{2}}-\frac{4}{1+2 \omega_{0} \bar{x}(t)^{2}}\right\}+ \\
& \frac{\omega_{0}}{1+2 \omega_{0} \bar{x}(t)^{2}}+\omega_{0}-\frac{\omega_{0}^{2} A^{2} \operatorname{Sin}^{2}(\omega t)}{2} \\
\beta(x, t)= & A \omega \operatorname{Cos}(\omega t) x-A^{2}\left(\omega^{2}-\frac{\omega_{0}^{2}}{2}\right) \frac{\operatorname{Sin}(2 \omega t)}{4 \omega}
\end{aligned}
$$

The phase $\beta(x, t)$ is uniquely determined up to a purely time-dependent function. At large $x$ both the potentials $v(\bar{x}, t), v^{\prime}(\bar{x}, t) \rightarrow \frac{\omega_{0}^{2} x^{2}}{2}+\lambda x \operatorname{Sin}(\omega t)$ and the quasienergy is $\tilde{\varepsilon}=\frac{3 \omega_{0}}{2}+\frac{A^{2}\left(\omega^{2}-\omega_{0}^{2} / 2\right)}{2} \operatorname{modulo}(\omega)$

Based on the fact that they have been able to generate one density from two different potentials, Maitra and Burke then conclude that this is a manifestation of the initial state-dependence of TDDFT. Thus they find Floquet theory of TDDFT to be incomplete. We now show that Maitra and Burke are incorrect on two counts. First, they have considered a system in steady-state solution that is supposed to have been obtained by an adiabatic switching. Thus RG theory is not applicable to this system. Therefore one cannot talk about the dependence on the initial state which is a specific feature of the RG theory. The problem falls in the domain of steady-state solutions and as such should be looked at within the Floquet theory of TDDFT. As discussed earlier, the domain of applicability of the Floquet theory of TDDFT is precisely the kind of example that has been considered above. Secondly, the Floquet theory of TDDFT is similar to the stationary-state DFT and as the derivation earlier shows, it is applicable to only the ground-states of the steady-states. Maitra and Burke on the other hand have applied it to an excited-state of the steady-state solution and shown that an excited-state density can be generated by two different potentials. That however, does not invalidate Floquet theory of TDDFT. This point needs further elaboration and we do that in the following.

For our discussion, we show that even in the stationary-state theory, we can generate 


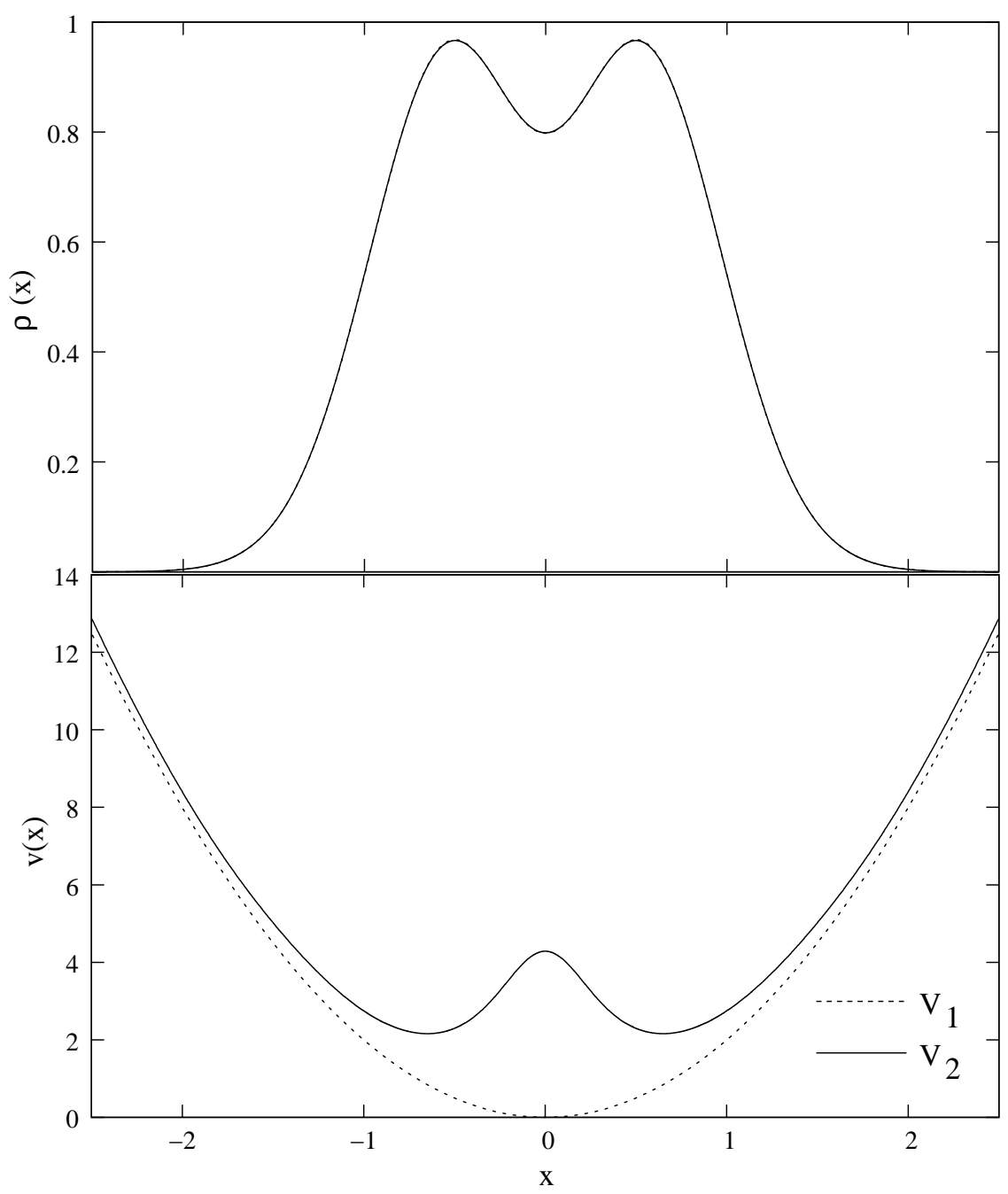

FIG. 1: Shown in the figure are the two potentials (lower panel) giving rise to the same excitedstate density (upper panel)of a harmonic 1D oscillator by putting one electron in the ground and other electron in the first excited-state.

a given excited-state density by two different potentials [7, 8, 9, 10, 11]. Again consider two electrons in a one-dimensional harmonic oscillator potential. If the two electrons are in the ground-state, the potential is determined uniquely by the Hohenberg-Kohn theorem. However, for excited-states, there is no such theorem and more than one potentials can give the same density. For this let us consider one electron in $n=0$ and one in $n=1$ state. The corresponding density is shown in the upper panel of Fig. 1. One potential corresponding to this density is obviously the harmonic potential $v(x)=\frac{1}{2} \omega_{0}^{2} x^{2}$ itself. Another potential can be generated by putting both the electrons in the ground-state orbital and then by inverting the Schrödinger equation or by using the van-Leeuwen-Baerends method [35]. 


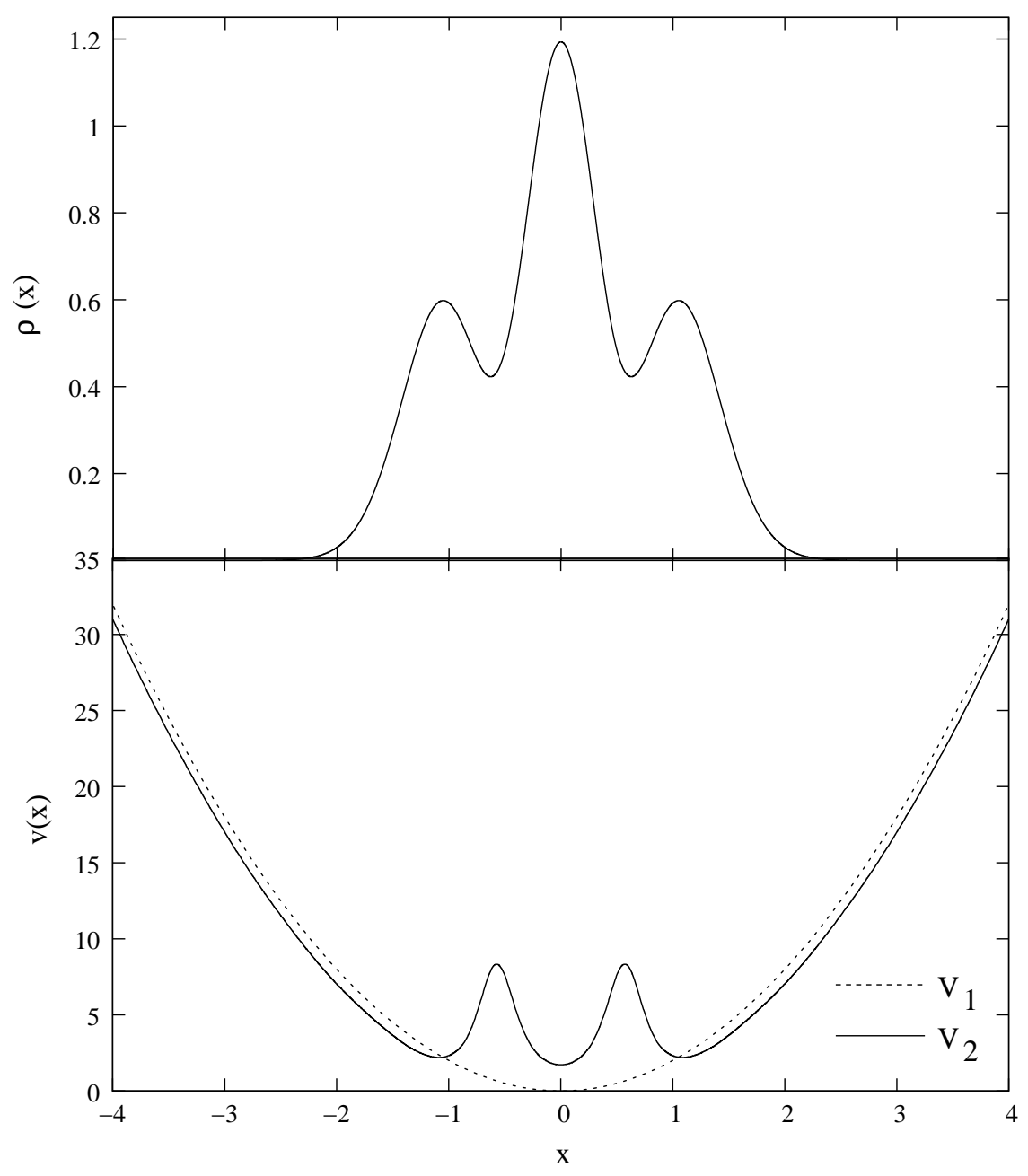

FIG. 2: Two potentials (lower panel) yielding the same excited-state density (upper panel) for an excited state of the two-electron in an 1D harmonic oscillator.

These two potentials are shown in the lower panel of Fig. 1. We consider one more example similar to that discussed above, where the excited-state density of two non-interacting electrons moving in a one-dimensional harmonic oscillator potential is obtained by putting one electron in $n=0$ and the other in $n=2$ state. Now this density (shown in the upper panel of Fig. 2) is produced by an alternative potential, which is shown in the lower panel of Fig. 2 along with the original potential. Thus if a system is in excited-state, more than one potentials can give the same density. On the other hand for the ground-state, occupation is uniquely defined and so is the potential. More examples of this kind exist in the literature [7, 8, 9, 10, 11]. Exactly in the same manner as in stationary-state theory, in Floquet theory of TDDFT also the "ground-state" gives the potential uniquely but for 
"excited-states" more than one potential can be found. This is precisely what has been done in [29]. Maitra and Burke do state in their work that their results resemble those of excited-states in time-independent problems but fail to make further connection with the latter.

Having explained the work of Maitra and Burke, we now also comment on the TDDFT calculations in light of the Floquet and RG theories of TDDFT. It is clear that in calculating frequency dependent polarizability $\alpha(\omega)$, it is the steady-state of a system that is employed. Thus in calculation of $\alpha(\omega)$, and related quantities such as excitation energies and oscillator strengths, [16] it is the Floquet theory of TDDFT rather than the RG theory that is being applied. Finally, one may raise a question if Floquet theory of TDDFT is applicable only to "ground-states". The answer is that even for steady-state "excited-states", a theory similar to the stationary-state excited-state DFT [4, 5, 6, 7, 8, 9, 10, 11] can be developed but that is not our main concern here.

To conclude, we have shown that Floquet theory of TDDFT is well founded and is distinct from the RG theory. Further, its recent criticism by Maitra and Burke is easily explained on the basis of a careful analysis of Floquet theory and RG theory.

Acknowledgement: We thank Professors B. M. Deb and K.D. Sen and Drs. S. K. Ghosh and Arup Banerjee for their comments on the manuscript. Critical remarks of Dr. Telnov are also acknowledged with pleasure. Discussions with Dr. K. Srihari are also acknowledged.

\section{APPENDIX A}

In this appendix, we show that if one confines the quasienergies to a range such that they go to their unperturbed counterpart when the time-periodic potential is turned off, then there is a well defined ground-state quasienergy satisfying the minimum variational principle. We first point out that defining the eigenenergies as suggested above is equivalent to taking the corresponding quasienergy state such that it has no free time-dependent factor 
of the form $\left(\sum_{p} e^{i p \omega t}\right)$, where $\{p=0, \pm 1, \pm 2, \ldots . \pm i, .$.$\} , associated with it as the strength$ of the time-periodic potential goes to zero. Let us denote such quasienergy states as $\phi_{0}(\vec{r}, t), \phi_{1}(\vec{r}, t), \ldots, \phi_{i}(\vec{r}, t), \ldots$ with the corresponding eigenvalues $\varepsilon_{0}, \varepsilon_{1}, \ldots, \varepsilon_{i}, \ldots$ such that $\varepsilon_{0}<\varepsilon_{1}<\ldots<\varepsilon_{i}<\ldots$. The quasienergy states satisfy the normalization condition

$$
\int \phi_{m}^{\dagger}(\vec{r}, t) \phi_{n}(\vec{r}, t) d \vec{r}=\delta_{m n}
$$

as is easily seen by the hermiticity of the Hamiltonian coupled with its adiabatic switching.

Let us consider a Hamiltonian $\hat{H}(\vec{r}, t)=\hat{H}_{0}(\vec{r})+\hat{v}(\vec{r}, t)$ with $\hat{v}(\vec{r}, t)$ being time periodic. Now a trial periodic function $\Phi(\vec{r}, t)$ can be expanded in terms of unperturbed states $\bar{\phi}_{i}(\vec{r})$ 's of $\hat{H}_{0}(\vec{r})$ and its associated states $\bar{\phi}_{i}(\vec{r}) e^{i p \omega t}$ as

$$
\begin{aligned}
\Phi(\vec{r}, t)= & a_{0} \bar{\phi}_{0}(\vec{r})+a_{1} \bar{\phi}_{1}(\vec{r})+\ldots .+a_{i} \bar{\phi}_{i}(\vec{r})+\ldots . \\
& +a_{0}^{(\omega)} \bar{\phi}_{0}(\vec{r}) e^{i \omega t}+a_{1}^{(\omega)} \bar{\phi}_{1}(\vec{r}) e^{i \omega t}+\ldots .+a_{i}^{(\omega)} \bar{\phi}_{i}(\vec{r}) e^{i \omega t}+\ldots . \\
& +a_{0}^{(2 \omega)} \bar{\phi}_{0}(\vec{r}) e^{i 2 \omega t}+a_{1}^{(2 \omega)} \bar{\phi}_{1}(\vec{r}) e^{i 2 \omega t}+\ldots .+a_{i}^{(2 \omega)} \bar{\phi}_{i}(\vec{r}) e^{i 2 \omega t}+\ldots . \\
= & \left(\sum_{p} a_{0}^{(p \omega)} e^{i p \omega t}\right) \bar{\phi}_{0}(\vec{r})+\left(\sum_{p} a_{1}^{(p \omega)} e^{i p \omega t}\right) \bar{\phi}_{1}(\vec{r})+\ldots . \\
& +\left(\sum_{p} a_{i}^{(p \omega)} e^{i p \omega t}\right) \bar{\phi}_{i}(\vec{r})+\ldots \ldots
\end{aligned}
$$

In the expansion above, each coefficient $a_{i}^{(p \omega)}(p \neq 0)$ should become zero as $\hat{v}(\vec{r}, t) \rightarrow 0$. Otherwise coefficient $a_{i}^{(p \omega)}$ would have two components: one arising from the applied timedependent potential causing unperturbed states to mix and the other is the coefficient of physically equivalent state $\phi_{i} e^{i p \omega t}$. To see this let us add $\Phi(\vec{r}, t) e^{i \omega t}$ to $\Phi(\vec{r}, t)$ to get

$$
\begin{aligned}
\Phi^{\prime}(\vec{r}, t)= & a_{0} \bar{\phi}_{0}(\vec{r})+a_{1} \bar{\phi}_{1}(\vec{r})+\ldots .+a_{i} \bar{\phi}_{i}(\vec{r})+\ldots . \\
& +\left(a_{0}^{(\omega)}+a_{0}\right) \bar{\phi}_{0}(\vec{r}) e^{i \omega t}+\left(a_{1}^{(\omega)}+a_{0}\right) \bar{\phi}_{1}(\vec{r}) e^{i \omega t}+\ldots .+\left(a_{i}^{(\omega)}+a_{0}\right) \bar{\phi}_{i}(\vec{r}) e^{i \omega t}+\ldots . \\
= & \left(1+e^{i \omega t}\right) \Phi(\vec{r}, t)
\end{aligned}
$$

For normalized wavefunctions the factor in front of $\Phi(\vec{r}, t)$ gives rise to a purely timedependent phase factor and can therefore be ignored. This is best illustrated if we look at the unperturbed time-independent problem in the Floquet formulation. 
In the case of time-independent problem in Floquet formulation the time-dependent wavefunction corresponding to a state $\phi_{i}(\vec{r})$ can be written in two equivalent forms as

$$
\Psi_{i}(\vec{r}, t)=e^{-i E_{i} t} \phi_{i}(\vec{r}) \equiv e^{-i(E+p \omega) t} \underbrace{\phi_{i}(\vec{r}) e^{+i p \omega t}=\phi_{i}(\vec{r}, t)},
$$

where $\phi_{i}(\vec{r}, t)$ are the Floquet states satisfies

$$
\left(\hat{H}(t)-i \frac{\partial}{\partial t}\right) \phi_{i}(\vec{r}, t)=\left(E_{i}+p \omega\right) \phi_{i}(\vec{r}, t)
$$

However, each $\phi_{i}(\vec{r}, t)$ gives the same $\Psi_{i}(\vec{r}, t)$. So they are all physically equivalent. If we were to expand a trial Floquet function in terms of $\left\{\phi_{i}(\vec{r})\right\},\left\{\phi_{i}(\vec{r}) e^{i \omega t}\right\}, \ldots .,\left\{\phi_{i}(\vec{r}) e^{i p \omega t}\right\}$, all it will do is give a complicated time-dependent phase factor in front of $\phi_{i}(\vec{r})$. How does this physical equivalence gets reflected variationally is discussed next.

Let us take a trial periodic wavefunction as [31] for the unperturbed problem as

$$
\Phi(\vec{r}, t)=\sum_{i p} a_{i}^{(p)} \phi_{i}(\vec{r}) e^{i p \omega t}
$$

with all $\phi$ 's and their equivalent states included. The quasienergy of the system is

$$
\begin{aligned}
& \frac{1}{T} \int\left\langle\Phi(\vec{r}, t)\left|\hat{H}(t)-i \frac{\partial}{\partial t}\right| \Phi(\vec{r}, t)\right\rangle d t \\
= & \frac{1}{T} \int \sum_{i p, j q} a_{i}^{(p) \star} \phi_{i}^{\star}(\vec{r}, t) e^{-i p \omega t}\left(E_{j}+q \omega\right) a_{j}^{(q)} \phi_{j}(\vec{r}, t) e^{i q \omega t} d \vec{r} d t \\
= & \sum_{i p, j q} \delta_{i j} \delta_{p q}\left(E_{j}+q \omega\right) a_{i}^{(p) \star} a_{j}^{(q)} \\
= & \sum_{i p}\left|a_{i}^{(p)}\right|^{2}\left(E_{i}+p \omega\right) .
\end{aligned}
$$

Since $\Phi(\vec{r}, t)$ is normalized at all times, to obtain $a_{i}^{(p)}$, the functional above is made stationary [26] with the condition

$$
\frac{1}{T} \int\langle\Phi(\vec{r}, t) \mid \Phi(\vec{r}, t)\rangle=\sum_{i p}\left|a_{i}^{(p)}\right|^{2}=1 .
$$

Using the techniques of Lagrange multipliers this leads to the equation

$$
a_{i}^{(p)}\left[E_{i}+p \omega-\mu\right]=0
$$


where $\mu$ is the Lagrange multiplier and gives the quasienergy of the system. The equation above gives $\mu=E_{i}+p \omega$ with $a_{i}^{(p)} \neq 0$ but all other $a_{i}^{(p)}$ 's vanishes. Thus stationary variational procedure picks Floquet states from only one particular zone (i.e. ' $p$ ' is fixed) to represent the system. However, irrespective of which ' $p$ ' we take, the final wavefunction is the same. Thus Floquet states from different zones do not represent different states. As such one may restrict oneself to only one zone in the expansion of the Floquet state. Question that arise is: does restricting oneself to one particular zone lead to a minimum principle? Actually, it does as we will now show. It is best shown for $p=0$ zone but the result is true for any ' $p$ '. Thus

$$
\begin{gathered}
\Phi(\vec{r}, t)=\sum_{i, p=0} a_{i}^{(0)} \phi_{i}(\vec{r}), \\
\left\langle\Phi\left|\hat{H}-i \frac{\partial}{\partial t}\right| \Phi\right\rangle=\sum_{i}\left|a_{i}^{(0)}\right|^{2} \varepsilon_{i}>\sum_{i}\left|a_{i}^{(0)}\right|^{2} \varepsilon_{0},
\end{gathered}
$$

by the standard variational argument.

Having discussed the time-independent case, we now discuss the time-dependent case. In this case the basis functions are the Floquet state $\phi_{i}(\vec{r}, t)$ of the full Hamiltonian and their physically equivalent counterparts $\phi_{i}(\vec{r}, t) e^{i p \omega t}$. If we expand a trial Floquet state $\Phi(\vec{r}, t)$ as

$$
\Phi(\vec{r}, t)=\sum_{i p} c_{i}^{(p)} \phi_{i}(\vec{r}, t) e^{i p \omega t},
$$

we again argue that including Floquet states from all zones does not really give us any new information. So the expansion should be restricted to only one zone. Mathematically it is again shown as was done above. The approximate quasienergy is given as

$$
\frac{1}{T} \int\left\langle\Phi\left|\hat{H}-i \frac{\partial}{\partial t}\right| \Phi\right\rangle d t=\sum_{i, p^{\prime}}\left|c_{i}^{(p)}\right|^{2}\left(\varepsilon_{i}+p \omega\right) .
$$

So the stationarity [26] of the time averaged expectation value in EqA13 under the constraint $\frac{1}{T} \int\langle\Phi \mid \Phi\rangle d t=1$ gives

$$
c_{i}^{(p)}\left\{\varepsilon_{i}+p \omega-E\right\}=0
$$


implying again that $E=\varepsilon_{i}+p \omega$ with $c_{i}^{(p)}$ - only in one zone. However, Floquet states from each zone give the same wavefunction $\Psi=e^{-i(E+p \omega) t} \phi_{i}(\vec{r}, t) e^{i p \omega t}$. Thus in expanding an approximate $\Phi(\vec{r}, t)$ one can restrict oneself to one particular zone. This leads to a minimum principle following the standard arguments.

The question arises how do we make sure that the trial wavefunction comprises Floquet states from one particular zone only. This is best done for $p=0$ zone by making sure that if we take $v(\vec{r}, t)=0$, the trial wavefunction must be time-independent. If Floquet states from other zones are also present in the trial wavefunction, it will not become time-independent as the time-dependent potential becomes zero. With such a trial wavefunction the quasienergy follow a minimum principle as shown above.

[1] P. Hohenberg and W. Kohn, Phys. Rev. 136 (1964) B864.

[2] W. Kohn and L. J. Sham, Phys. Rev. 140 (1965) A1133 .

[3] R.G. Parr and W. Yang, Density Functional Theory of Atoms and Molecules, Oxford Univ. Press, New York, 1989 .

[4] A. Görling, Phys. Rev. A 54 (1996) 3912.

[5] A. Görling, Phys. Rev. A59 (1999) 3359.

[6] M. Levy and Á. Nagy, Phys. Rev. Lett. 83 (1999) 4361;

Á. Nagy and M. Levy, Phys. Rev. A 63 (2001) 052502.

[7] V. Sahni, L. Massa, R. Singh and M. Slamet, Phys. Rev. Lett. 87 (2001) 113002 .

[8] M. K. Harbola, Phys. Rev. A 69 (2004) 042512.

[9] R. Gaudoin and K. Burke, Phys. Rev. Lett. 93 (2004) 173001 ; ibid. 94 (2005) 029901.

[10] P. Samal, M. K. Harbola and A. Holas, Chem. Phys. Lett. 419 (2006) 217 ; ibid. 422 (2006) 586 .

[11] P. Samal and M. K. Harbola, J. Phys. B 39 (2006) 4065 .

[12] B.M. Deb, S.K. Ghosh, J. Chem. Phys. 77 (1982) 342 .

[13] L.J. Bartolotti, Phys. Rev. A 24 (1981) 1661.

[14] E. Runge, E.U.K. Gross, Phys. Rev. Lett. 52 (1984) 997.

[15] X.-M. Tong and S.-I. Chu, Phys. Rev. A 57 (1998) 452 . 
[16] M.E. Casida, Recent Advances in Density Functional Methods, Part 1, edited by D.P. Chong, World Scientific, Singapore, 1995 .

[17] M. Petersilka,U.J. Gossmann, and E.K.U. Gross, Phys. Rev. Lett. 76 (1996) 1212 .

[18] S. van Gisbergen, et al., J. Chem. Phys. 103 (1995) 9347.

[19] F. Nogueira, M. A. L. Marques and C. Fiolhais, A Primer in Density Functional Theory, Springer Verlag, 2003 .

[20] H. Rabitz, Science 292 (2001) 709 .

[21] S.-I. Chu, Adv. At. Mol. Phys. 21 (1985) 197.

[22] S.-I. Chu, Adv. Chem. Phys. 73 (1989) 739 .

[23] D. A. Telnov, S.-I. Chu, Chem. Phys. Lett. 264 (1997) 466 .

[24] D. A. Telnov and S.-I. Chu, Phys. Rev. A 58 (1998) 4749 .

[25] S.-I. Chu and D. A. Telnov, Physics Reports 390 (2004) 1 .

[26] M. K. Harbola and A. Banerjee, Ind. J. Chem. 39A (2000) 9 .

[27] N.T. Maitra, K. Burke, Phys. Rev. A 63 (2001) 042501 ; 64 (2001) 039901 (E).

[28] R. van Leeuwen, Phys. Rev. Lett. 82 (1999) 3863.

[29] N. T. Maitra and K. Burke Chem. Phys. Lett. 359 (2002) 237.

[30] M. Krykunov and J. Autschbach, J. Chem. Phys. 123 (2005) 114103.

[31] J.H. Shirley, Phys. Rev. 138 (1965) B979 .

[32] H. Sambe, Phys. Rev. A 7 (1973) 2203.

[33] P.W. Langhoff, S.T. Epstein, M. Karplus, Rev. Mod. Phys. 44 (1972) 602 .

[34] H.P. Breuer, M. Holthaus, Z. Phys. D 11 (1989) 1 ; Ann. Phys. 214 (1991) 249.

[35] R. van Leeuwen, E. J. Baerends, Phys. Rev. A 49 (1994) 2421. 